\title{
Perforation and Bacterial Contamination of Microscope Covers in Lumbar Spinal Decompressive Surgery
}

\author{
Georg Osterhoff $^{a}$ José Spirig ${ }^{a} \quad$ Jürgen Klasen ${ }^{a}$ Stefan P. Kuster ${ }^{b}$ \\ Annelies S. Zinkernagel ${ }^{\mathrm{b}}$ Hugo Sax ${ }^{\mathrm{b}} \mathrm{Kan} \mathrm{Min}^{\mathrm{a}}$ \\ ${ }^{a}$ Department of Orthopedics, Balgrist Clinic, University of Zurich, and ${ }^{b}$ Division of Infectious Diseases and Hospital \\ Epidemiology, University Hospital Zurich, University of Zurich, Zurich, Switzerland
}

\section{Key Words}

Surgical infection · Operation microscope · Spine .

Decompression

\begin{abstract}
Objective: To determine the integrity of microscope covers and bacterial contamination at the end of lumbar spinal decompressive surgery. Materials and Methods: A prospective study of 25 consecutive lumbar spinal decompressions with the use of a surgical microscope was performed. For detection of perforations, the microscope covers were filled with water at the end of surgery and the presence of water leakage in 3 zones (objective, ocular and control panel) was examined. For detection of bacterial contamination, swabs were taken from the covers at the same locations before and after surgery. $\boldsymbol{R} \boldsymbol{e}$ sults: Among the 25 covers, 1 (4\%) perforation was observed and no association between perforation and bacterial contamination was seen; 3 (4\%) of 75 smears from the 25 covers showed post-operative bacterial contamination, i.e. 2 in the ocular zone and 1 in the optical zone, without a cover perforation. Conclusions: The incidence of microscope cover perforation was very low and was not shown to be associated with bacterial contamination. External sources of bacterial contamination seem to outweigh the problem of contamination due to failure of cover integrity.

๑) 2014 S. Karger AG, Basel
\end{abstract}

\section{Introduction}

Surgical site infections are reported to account for $38 \%$ of all surgical nosocomial infections [1]. They are associated with increased costs and lengths of hospital stay [2-4]. Seventy-seven per cent (77\%) of acute mortalities in surgical patients with surgical site infections have been found to be infection related [1]. Post-operative surgical site infections have been shown to occur in $1-17 \%$ of cases [4-7] after spinal surgery. Several factors like diabetes mellitus, disseminated cancer, obesity, smoking, spinal fusion and the duration of surgery have been reported to be associated with an increased rate of surgical site infection in spinal surgery $[4,8,9]$. In an attempt to minimize the potential sources of surgical site infections, recent studies have pointed out the possible role of bacterial shedding by operation microscopes [10-12]. Bacterial contamination of the microscope cover was shown to be present in $12-44 \%$ of the samples taken at different sites of the cover $[10,12]$.

However, it has not been possible to determine if the cover contamination was caused by an external source (i.e. direct external contact like the surgeon's head or mask) or derived internally from the microscope itself. Thin sterile plastic foil covers are used universally as microscope covers. In the case of arthroscopic camera covers in orthopaedic surgery, it was shown that these foils are prone to dam-

\begin{tabular}{ll}
\hline KARGER & $\begin{array}{l}\text { () 2014 S. Karger AG, Basel } \\
1011-7571 / 14 / 0234-0302 \$ 39.50 / 0 \quad \text { Karger }\end{array}$ \\
$\begin{array}{l}\text { E-Mail karger@karger.com } \\
\text { www.karger.com/mpp }\end{array}$ & $\begin{array}{l}\text { This is an Open Access article licensed under the terms of the } \\
\text { Creative Commons Attribution-NonCommercial 3.0 Un- } \\
\text { ported license (CC BY-NC) (www.karger.com/OA-license), } \\
\text { applicable to the online version of the article only. Distribu- } \\
\text { tion permitted for non-commercial purposes only. }\end{array}$
\end{tabular}

Georg Osterhoff, MD

Department of Orthopedics

Balgrist Clinic, University of Zurich

Forchstrasse 340, CH-8008 Zurich (Switzerland)

E-Mail georg.osterhoff@usz.ch 


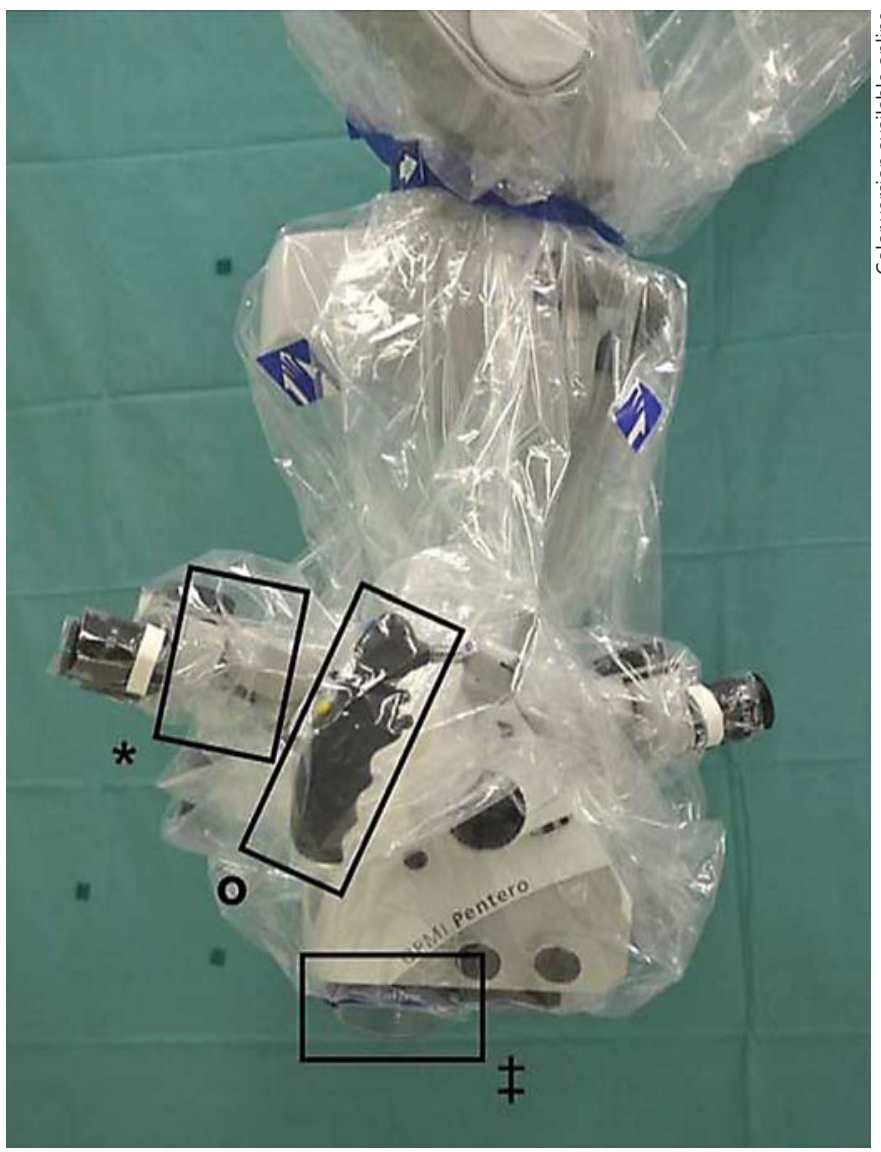

Fig. 1. Zones for microbiological smears. * Main (surgeon's) ocular cover. ${ }^{\ddagger}$ Objective. ${ }^{\circ}$ Control panel.

age by manipulations during the surgical procedure, resulting in small defects of the foil [13]. This would allow contamination from the microscope itself. We presumed that small holes could also appear in microscope covers as a result of manipulation of the microscope which may lead to bacterial contamination of the surgical field. To our knowledge, this possible complication has not been studied systematically. Therefore, the aim of this study was to determine the integrity of microscope covers after spinal surgery and to assess the possible relationship between the incidence of cover perforations and the occurrence of bacterial contamination of the microscope covers.

\section{Materials and Methods}

A prospective study of consecutive spinal interventions with the use of a surgical microscope was performed. Plastic covers from 2 microscopes (M680; Leica Microsystems GmbH, Wetzlar, Germany, and OPMI Pentero; Carl Zeiss AG, Oberkochen, Germany) that

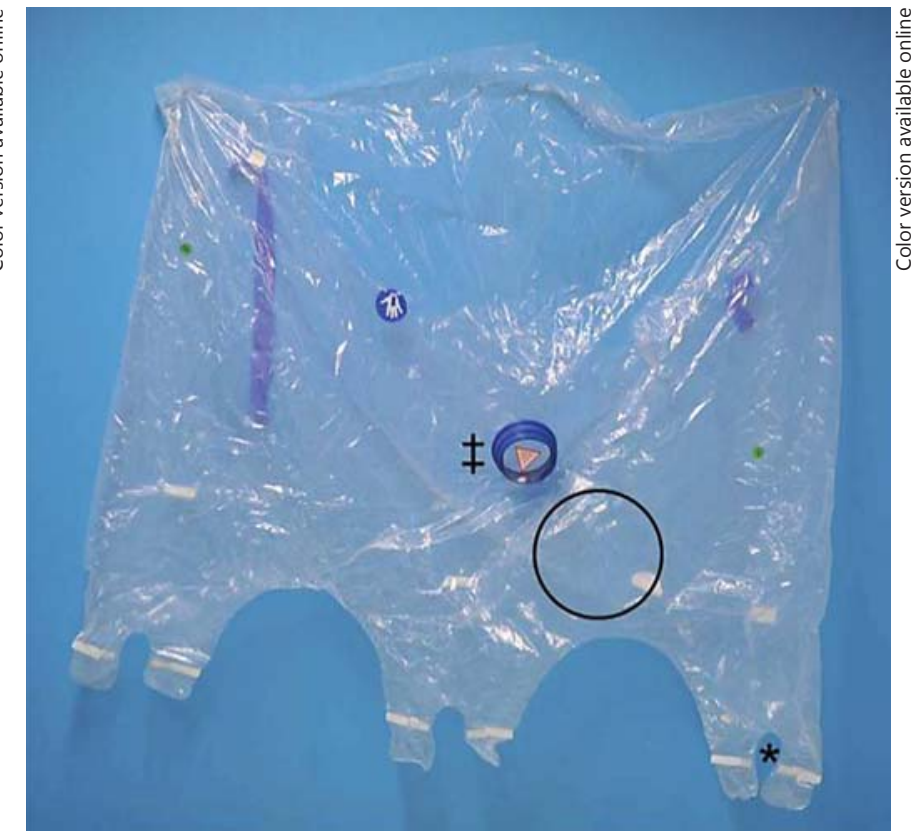

Fig. 2. Part of the microscope cover as removed after surgery. ${ }^{*}$ Ocular cover. ${ }^{\ddagger}$ Objective. ${ }^{\circ}$ Foil covering the control panel.

were used during spinal surgery were collected $(\mathrm{n}=25 ; \mathrm{M} 680: \mathrm{n}=$ 2, OPMI Pentero: $n=23$ ). As recommended by the manufacturers, Wild Leica 137/380 covers were used for the Leica microscope and OPMI Drapes Carl Zeiss covers were used for the Zeiss microscope. Covers were draped by a scrub nurse and the operating surgeon together, and both followed the standard aseptic technique. Gloves were changed after fitting of the lens cover. This study was conducted in compliance with the Helsinki Declaration and in accordance with the guidelines of the institutional ethics committee.

\section{Patients/Intervention}

Twenty-five lumbar spinal decompression procedures in 25 patients from October 2012 to December 2012 were prospectively included in this study. A sample size of 25 covers was chosen as a previous study on arthroscopic covers reported a perforation rate of 0.74 , suggesting a probability of 0.0005 of observing no tears in 25 covers [13]. The involved doctors were experienced spinal surgeons who had performed over 200 spinal surgeries per year with the use of a surgical microscope at the authors' institution. Intravenous cefazolin was given as an antibiotic prophylaxis $30 \mathrm{~min}$ before skin incision. Surgery was performed in a laminar airflow operating facility.

\section{Assessment of Microscope Cover Contamination}

In order to check for contamination originating from external sources (i.e. direct contact with the surgeon's head or mask), bacterial contamination of the microscope covers was assessed after encasing the microscopes. Microbiological smears were taken from each of the covers divided as described above [objective zone, main (surgeon's) ocular zone and control panel zone] (fig. 1) before the start of the surgical procedure. After the surgery and before remov- 
Fig. 3. Cover integrity testing setup. Ocular part of the cover filled with water. a No perforation. $\mathbf{b}$ Water leakage due to a perforation $\leq 1 \mathrm{~mm}$ in diameter.
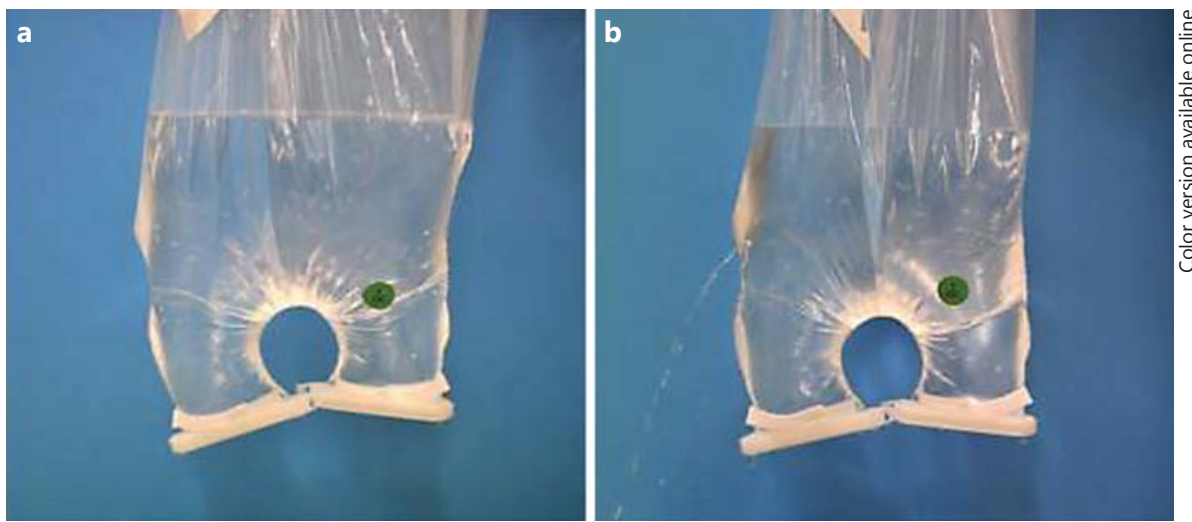

al of the covers, smears were taken at the same locations again. To serve as a positive control, 2 smears were taken from each zone of an uncovered microscope. All smears were obtained using sterile culture swabs (eSwab; COPAN, Brescia, Italy). In a blinded manner, the swab was subsequently assessed using a standard semiquantitative technique in laminar flow hoods. Swabs were streaked on one third of a $5 \%$ sheep blood Columbia agar plate (BioMérieux, Geneva, Switzerland) and then placed in Luria Bertani broth (Sigma) for enrichment for $72 \mathrm{~h}$. With a disposable sterile loop, 2 successive dilutions were obtained by further streaking the initial inoculum on the agar plates over the remaining two thirds in succession, using a new loop for each streaking manoeuver. The plates were then incubated at $37^{\circ} \mathrm{C}$ for $48 \mathrm{~h}$, after which they were assessed for colony growth. After $72 \mathrm{~h}$, the liquid enrichment medium was processed on agar plates identical to the agar plate method described above. Gram stains were used for morphology and Gram classification. Bacteria were assessed for catalase reactivity and staphylococcal agglutination. Some subcultures were further identified using the RapID Staph Plus System (BioMérieux).

\section{Assessment of Microscope Cover Integrity}

In order to check for internal contamination originating directly from the microscope itself, the foil integrity of the microscope covers was assessed. Immediately after surgery, the cover was cut circumferentially just above the strap closest to the microscope head and the cover was then carefully removed from the microscope (fig. 2). All covers were tested for perforations. Preexisting openings for the oculars and the objective were closed water-tight with plastic clamps before testing. The covers were divided into 3 zones: an objective zone, a main (surgeon's) ocular zone and a control panel zone (fig. 1,2). The covers were fixed in a custom-made testing device and the parts belonging to the zones mentioned above were filled with water up to a level of $25 \mathrm{~cm}$. The presence of water leaks and their localizations were documented (fig. 3). As previously described for arthroscopic covers [13], holes in the plastic covers were classified as small (identified by water leakage but with no hole visible to the human eye), medium (hole $\leq 1 \mathrm{~mm}$ in diameter) and large (hole $>1 \mathrm{~mm}$ in diameter).

\section{Statistical Analysis}

To detect relationships between the occurrence of perforations or bacterial contaminations and the duration of surgery, the latter
Table 1. Duration of surgery

\begin{tabular}{lllcl}
\hline & & \multicolumn{2}{l}{ Duration } & Total \\
\cline { 3 - 4 } & & $<1 \mathrm{~h}(\mathrm{n}=6)$ & $>1 \mathrm{~h}(\mathrm{n}=19)$ & \\
\hline Perforation $^{\mathrm{a}}$ & yes & 0 & 1 & 1 \\
& no & 6 & 18 & 24 \\
\hline Postoperative $_{\text {contamination }^{\mathrm{b}}}$ & yes & 0 & 3 & 3 \\
\hline
\end{tabular}

${ }^{\mathrm{a}}$ Fisher's exact test: $\mathrm{p}=1 .{ }^{\mathrm{b}}$ Fisher's exact test: $\mathrm{p}=0.554$.

was divided into durations $\leq 1 \mathrm{~h}$ and $>1 \mathrm{~h}$. Nominal variables were then were associated in crosstabs with Fisher's exact tests using SPSS for Windows 17.0 (SPSS, Chicago, Ill., USA). p < 0.05 was considered statistically significant.

\section{Results}

The mean duration of the operations was $89 \pm 31 \mathrm{~min}$ (range 48-153). No perioperative complications were observed; in particular, there was no post-operative surgical site infection.

\section{Bacterial Microscope Cover Contamination}

Of the 25 covers, 3 (12\%) showed post-operative bacterial contamination: 2 (8\%) in the ocular zone and $1(4 \%)$ in the objective zone. No significant relationship was detected between duration of surgery $>1 \mathrm{~h}$ and presence of a post-operative bacterial contamination (table $1 ; \mathrm{p}=0.554$ ).

Though serving as a negative control, 6 of 25 covers showed pre-operative contamination [2 each in the ocular, control panel and objective zones; i.e. $6 / 75$ (8\%) pre- 
operative smears] that was not reproducible post-operatively. Six of 6 positive controls taken directly from the uncovered microscope were positive.

Most isolated microorganisms (pre-operative, postoperative and control) were coagulase-negative staphylococci (Staphylococcus capitis and S. epidermidis). In 2 preoperative smears ( 1 in the ocular zone and 1 in the objective zone), Micrococcus species were detected. In all cases, growth was only seen after enrichment, implying bacterial concentrations of 1-100 microorganisms $/ \mathrm{ml}$. No bacteria were detected when applying the swabs directly on agar.

\section{Microscope Cover Integrity}

Of the 25 covers, 1 (4\%) perforation $\leq 1 \mathrm{~mm}$ in diameter in the control panel area was observed. This cover showed no bacterial contamination post-operatively. No significant relationship was detected between duration of surgery $>1 \mathrm{~h}$ and presence of a perforation (table $1 ; \mathrm{p}=1$ ).

\section{Discussion}

Only 1 small perforation in 1 of the 25 covers was observed, which was, however, not associated with bacterial contamination. The removal procedure could have caused the lesion. Three of the 75 smears from a total 25 covers showed post-operative bacterial contamination, i.e. 2 in the ocular zone and 1 in the objective zone, without proof of a cover perforation. These numbers must be interpreted with caution, though, as 6 of the 75 pre-operative smears (negative control) were positive as well. The latter implies a possible high rate of false positives for all smears. In contrast, as all smears that were taken directly from the uncovered microscope (positive control) were positive, the rate of false negatives was low.

It is therefore unlikely that the bacterial contamination of the covers was facilitated through lesions in the cover foil. Apparently, this finding contradicts the hypothesis of internal contamination of the covers and supports the hypothesis of an external source of bacterial contamination (i.e. the surgeon or the nurse). The $4 \%$ rate of contamination in our study was smaller than those published previously (12-20\% bacterial contamination of microscope covers) [10-12]. Tronnier et al. [11] reported contamination of the lens cover in $12 \%$ of cases and Bible et al. [12] reported contamination rates of $12-20 \%$ in each of the areas that are comparable to our study. The study by Tronnier et al. [11] was conducted 25 years ago and used different covers with different sterility standards, which might explain the higher bacterial contamination rate. Bible et al. [12] used OPMI Drapes (Carl Zeiss AG), as in the vast majority of our cases. In contrast to our study, however, they performed their interventions in a non-laminar flow operating room. It remains unclear whether a laminar flow reduces the bacterial density in the air near the operation field. Two of 3 post-operative cover contaminations were observed in the ocular zone, which are most likely explained by shedding of dander from the face or by accidental touching of the unsterile area when adjusting the ocular focus during surgery [12].

There is no clear explanation for the high rate of preoperative bacterial contamination of the covers. Postsampling contamination during processing of the swabs is unlikely, as this was performed in a certified laboratory with an appropriate infrastructure (e.g. laminar flow hoods). Post-sampling contamination in the operation room is possible but would not explain the asymmetric (pre-/post-operative) distribution of bacterial growth.

Nevertheless, the bacterial burden was very low since bacteria were only detected after using enrichment media. In addition, the rate of contamination was smaller than in previous studies on bacterial contamination of microscope covers [10-12].

True contamination due to impaired sterility provided by the manufacturer or incorrect fitting of the cover is unlikely as we can assume a good sensitivity of the microbiological smears (all positive controls were positive) and bacterial growth was not reproducible in the same regions post-operatively in any of the cases.

The use of microscopes in spinal surgery has become standard. To date, there is no evidence of a superiority of clinical outcomes with the use of a microscope compared to the 'open' traditional technique. Future studies should be directed towards improving the design of these microscopes. As this study found external sources to be the main reason for bacterial contamination, new microscopes with integrated monitor systems [14] and touch screen technology might reduce potential causes of contamination. These technologies would decrease the amount of uncontrolled contact between the surgeon and the microscope cover. Knowledge about the source of bacterial contamination of foil covers is also important for designing covers for other equipment used in the intra-operative setting (e.g. portable CT scanners [15]).

A limitation of this study is that its prospective nature possibly introduced the problem of an observer effect. In this case, perforations might have been prevented because the study increased the attention of the surgical team. It is the authors' conviction, however, that surgeons 
using operative microscopes are always careful in their handling. No smears were taken from the 'overhead' area of the microscope covers that had been found to be prone to contamination during surgery [12], probably because this is mainly a problem of draping the cover and microscopes with air evacuation to remove the air between the microscope and the drape, which already have been suggested. Another limitation of this study resides in the fact that no post-operative surgical site infections were observed. Thus, the link between infection and microscope cover contamination cannot be proven or even evaluated by this study. An association between the use of operative microscopes and bacterial shedding into the operation field, however, has been reported in the literature before [10].

\section{Conclusions}

The incidence of microscope cover perforation as well as bacterial contamination was very low. The occurrence of cover perforation was not shown to be associated with bacterial contamination. External sources of bacterial contamination seem to outweigh the problem of contamination due to failure of cover integrity.

\section{Acknowledgement}

We would like to thank Massimo Leonardi for technical assistance.

\section{References}

1 Mangram AJ, Horan TC, Pearson ML, et al: Guideline for prevention of surgical site infection: Centers for Disease Control and Prevention (CDC) Hospital Infection Control Practices Advisory Committee. Am J Infect Control 1999;27:97-132, quiz 133-134, discussion 196.

-2 Poulsen KB, Bremmelgaard A, Sorensen AI, et al: Estimated costs of postoperative wound infections: a case-control study of marginal hospital and social security costs. Epidemiol Infect 1994;113:283-295.

3 Vegas AA, Jodra VM, Garcia ML: Nosocomial infection in surgery wards: a controlled study of increased duration of hospital stays and direct cost of hospitalization. Eur J Epidemiol 1993;9:504-510.

4 Veeravagu A, Patil CG, Lad SP, et al: Risk factors for postoperative spinal wound infections after spinal decompression and fusion surgeries. Spine (Phila Pa 1976) 2009;34: 1869-1872.
5 Olsen MA, Nepple JJ, Riew KD, et al: Risk factors for surgical site infection following orthopaedic spinal operations. J Bone Joint Surg Am 2008;90:62-69.

6 Roberts FJ, Walsh A, Wing P, et al: The influence of surveillance methods on surgical wound infection rates in a tertiary care spinal surgery service. Spine (Phila Pa 1976) 1998; 23:366-370.

7 Kanayama M, Hashimoto T, Shigenobu K, et al: Effective prevention of surgical site infection using a centers for disease control and prevention guideline-based antimicrobial prophylaxis in lumbar spine surgery. J Neurosurg Spine 2007;6:327-329.

-8 Wimmer C, Gluch H, Franzreb M, et al: Predisposing factors for infection in spine surgery: a survey of 850 spinal procedures. J Spinal Disord 1998;11:124-128.

$\checkmark 9$ Lamloum SM, Mobasher LA, Karar AH, et al: Relationship between postoperative infectious complications and glycemic control for diabetic patients in an orthopedic hospital in Kuwait. Med Princ Pract 2009;18:447-452.

10 Weiner BK, Kilgore WB: Bacterial shedding in common spine surgical procedures: headlamp/loupes and the operative microscope. Spine (Phila Pa 1976) 2007;32:918-920.
11 Tronnier V, Schneider R, Kunz U, et al: Postoperative spondylodiscitis: results of a prospective study about the aetiology of spondylodiscitis after operation for lumbar disc herniation. Acta Neurochir (Wien) 1992;117: 149-152.

12 Bible JE, O’Neill KR, Crosby CG, et al: Microscope sterility during spine surgery. Spine (Phila Pa 1976) 2012;37:623-627.

13 Werner CM, Necas T, Schneeberger AG: Defects of camera covers after arthroscopic surgery. J Shoulder Elbow Surg 2006;15:199-202.

14 Shirzadi A, Mukherjee D, Drazin DG, et al Use of the video telescope operating monitor (VITOM) as an alternative to the operating microscope in spine surgery. Spine (Phila Pa 1976) 2012;37:E1517-E1523.

15 Dohrmann GJ, Byrne RW: What's new in neurosurgery: advances in neurovascular and spine surgery, epilepsy surgery, surgery for movement disorders and intraoperative imaging. Med Princ Pract 2010;19:328-329. 